\title{
Gobernanza y universidad: estudio iberoamericano sobre la participación estudiantil en las instituciones de Educación Superior
}

\author{
Isaac Calduch (D) @ \\ Juan Llanes (1) @ \\ Alejandra Montané (1) @ \\ Jorge Luis Méndez-Ulrich (D) @ \\ Universitat de Barcelona (UB), España.
}

Resumen. El interés en la naturaleza de la participación estudiantil en la gobernanza y vida de la universidad se construye sobre los principios de democracia, autonomía y participación. En el texto se profundiza sobre los modos de participación estudiantil y los factores inhibidores del mismo. Se muestran y detallan los resultados de una investigación en la que participaron 13939 estudiantes de grados, másteres y programas de doctorado relacionados con el ámbito de la educación de 18 instituciones pertenecientes principalmente a Colombia, Argentina, Honduras, México, Ecuador, Bolivia, Paraguay, Portugal y España, dentro del contexto del proyecto TO-INN de cooperación interuniversitaria. Mediante una investigación cuantitativa por encuesta, se han obtenido resultados sobre los modos de participación estudiantil en las Instituciones de Educación Superior, la relación entre los modos de participación y las principales variables sociodemográficas (género, edad, discapacidad, pertenencia a comunidades indígenas, tener personas a su cargo y nivel formativo familiar). Finalmente se plantea el debate y las conclusiones sobre los niveles y modos de participación y la relevancia de la participación estudiantil en la gobernanza universitaria. Palabras clave: educación superior; participación estudiantil; gobernanza universitaria; dimensión social; estudiantes universitarios.

\section{Governança e universidade: estudo ibero-americano sobre a participação estudantil nas Insti- tuições de Ensino Superior}

Resumo. O interesse na natureza da participação estudantil na governança e vida da universidade é construído sobre os princípios de democracia, autonomia e participação. No texto, reflete-se sobre os modos de participação estudantil e seus fatores inibidores. Mostram-se e detalham-se os resultados de uma pesquisa em que participaram 13939 estudantes de graduação, mestrados e programas de doutorado relacionados com a área da educação de 18 instituições pertencentes principalmente a Colômbia, Argentina, Honduras, México, Equador, Bolívia, Paraguai, Portugal e Espanha, dentro do contexto do projeto TO-INN de cooperação interuniversitária. Mediante uma pesquisa quantitativa, obtiveram-se resultados sobre os modos de participação estudantil nas Instituições de Ensino Superior, a relação entre os modos de participação e as principais variáveis sociodemográficas (gênero, idade, deficiência, pertinência a comunidades indígenas, ter pessoas sob responsabilidade e nivel de formação familiar). Finalmente propõe-se o debate e as conclusões sobre os níveis e os modos de participação e a relevância da participação estudantil na governança universitária.

Palavras-chave: ensino superior; participação estudantil; governança universitária; dimensão social; estudantes universitários.

\section{Governance and University: Ibero-american study of student participation in Higher Education Institutions}

Abstract. The interest in the nature of student participation in governance and life of the university is built on the principles of democracy, autonomy and participation. The text delves into the modes of student participation and the factors that inhibit it. It shows and details the results of a study with 13939 students of undergraduate, postgraduate and doctoral programs related to the field of educationin 18 institutions, mainly from Colombia, Argentina, Honduras, Mexico, Ecuador, Bolivia, Paraguay, Portugal and Spain, in the context of the TO-INN project, an inter-university cooperation project. Through a survey-study, results have been obtained on the modes of student participation in the Higher Education Institutions, the relationship between participation modes and the main sociodemographic variables (age, gender, belonging to indigenous communities, dependenceand families'education level). Finally, the debate and conclusions on the levels and modes of participation and the relevance of student participation in university governance are presented.

Keywords: higher education; student participation; university governance; social dimension; college students. 


\section{Introducción}

EI proyecto TO-INN, From Tradition to Innovation in Teacher Training Institutions (De la tradición a la innovación en instituciones de formación de maestros), ${ }^{1}$ fue una investigación internacional desarrollada entre el año 2016 y 2019. Su objetivo principal era fomentar la innovación organizativa y pedagógica en las instituciones de Educación Superior participantes, desde un enfoque sistémico, fortaleciendo la innovación docente, la pertinencia curricular y la dimensión social, mediante la participación de todo el conjunto de la comunidad universitaria para impactar en las políticas y la gobernanza institucional.

Los lineamientos estratégicos que fundamentaron el proyecto quedan recogidos en la declaración de la // Conferencia Mundial sobre la Educación Superior (UNESCO, 2009), donde se reafirma la Educación Superior como un bien público, cuyo acceso constituye un derecho humano universal. En dicha declaración también se enfatiza la responsabilidad social, la equidad y la calidad como aspectos centrales para lograr que la Educación Superior en el siglo XXI sea motor del cambio social y el desarrollo sostenible. En esta misma línea, recientemente, en el cuarto objetivo de desarrollo sostenible de la Agenda 2030 de la Organización de las Naciones Unidas, aparece la meta de "asegurar el acceso en condiciones de igual para todos los hombres y las mujeres a una formación técnica, profesional y superior de calidad, incluida la enseñanza universitaria" (ONU, 2018), lo que supone de nuevo un marco de acción que insta a los diferentes países a ampliar el acceso, la equidad, la calidad y la pertinencia de la educación superior, contribuyendo así a lograr un desarrollo sostenible global.

Por otro lado, cabe añadir que este estudio se circunscribió a aqueIlas instituciones de Educación Superior que forman a los futuros maestros u otros profesionales de la Educación. Esto supone no solo unas propuestas curriculares distintivas, sino también una cultura académica idiosincrática. Esta puntualización es relevante, ya que los procesos de gobernanza universitaria no pueden ser ajenos a las distintas culturas académicas que conforman la institución y a las características propias de los actores de cada una de ellas. Por tanto, la elección de esta subpoblación universitaria no fue fortuita, sino que pretendía dar respuesta, de forma simultánea, a otros de los grandes

${ }^{1}$ From Tradition to Innovation in Teacher Training Institutions (573685-EPP2016-1-ES-EPP KA2-CBHE-JP). Proyecto financiado por la Unión Europea, en el marco del programa ERASMUS+: Key Action 2 - Capacity Building in the field of Higher Education (2016-2019). El consorcio de participantes estaba integrado por 22 instituciones pertenecientes a 11 países. 
retos educativos del contexto iberoamericano: la necesidad de fortalecer la profesión docente y mejorar la formación inicial del profesorado de Educación Primaria y Secundaria (OEI, 2010).

Para lograr la modernización de las Instituciones de Educación Superior es necesario adoptar un enfoque multidimensional. La reforma no puede entenderse mediante acciones puntuales y aisladas de innovación docente o de cambio curricular, sino que se debe apostar por una articulación más profunda de los distintos componentes que conforman las instituciones universitarias. En este sentido, el proyecto trabaja con un enfoque sistémico alrededor de cuatro ejes que vertebran el proceso de modernización de las Instituciones de Educación Superior: la innovación docente, la pertinencia curricular, su dimensión social y la gobernanza universitaria. Sin embargo, estos no deben concebirse como componentes independientes, sino en un constante proceso de interacción dinámica y de influencia mutua.

- La innovación docente consiste en la mejora de los procesos de enseñanza-aprendizaje y de la práctica educativa que acontece en las instituciones universitarias. Dicho proceso de mejora no debe ser entendido como el resultado de planes o proyectos puntuales de innovación, sino como un proceso de aprendizaje y cambio institucional, sostenido y sostenible en el tiempo, que debe enraizarse en la propia dinámica institucional mediante un tejido de creencias y significados compartidos, discursos, sistemas de relaciones y de influencias sociales, a la vez que prácticas concretas.

- La pertinencia curricular hace referencia a la adecuación de las titulaciones universitarias y de sus propuestas curriculares con las expectativas, necesidades y demandas de la sociedad (UNESCO, 1998, 2009). Una propuesta curricular es pertinente cuando es sensible, en su diseño y en su desarrollo, tanto a los intereses y demandas de los diversos contextos sociales, culturales y productivos en los que se inserta su institución (pertinencia sociocultural) como a las necesidades de aprendizaje y de desarrollo personal e intelectual de los estudiantes (pertinencia pedagógica).

- La dimensión social se enmarca en una visión humanista de la educación como bien común esencial (UNESCO, 2015), y puede sintetizarse en poner la universidad al servicio de la sociedad. La dimensión social de la educación superior significa un compromiso con la calidad, la inclusión, la equidad y la participación de la comunidad universitaria (especialmente la participación estudiantil), junto con la responsabilidad por el 
desarrollo sostenible y el liderazgo social. En este sentido, se aboga por una visión amplia de la noción de dimensión social, que vaya más allá del enfoque tradicional focalizado en la composición de la matrícula universitaria, concibiéndola juntamente con la tercera misión de la universidad y la Responsabilidad Social Universitaria. Sin embargo, esto no excluye que uno de los principales retos de la dimensión social de la universidad siga siendo la democratización del conocimiento y la equidad participativa (Ariño, 2014; Londres, 2007).

- La gobernanza universitaria se puede definir, bajo la óptica del proyecto TO-INN, como el "conjunto de procesos de gestión que incluyen modos de gobierno de la institución universitaria orientados por los principios que rigen una sociedad democrática y solidaria, con la finalidad de conseguir la mejora institucional y el desarrollo personal, académico y social de todos sus actores" (Antúnez, 2019, p.5). En este sentido, la gobernanza universitaria es entendida como un modo peculiar de gestionar las instituciones de educación superior bajo una serie de principios concretos, entre los que destacan la democracia, la autonomía y la participación.

En definitiva, la investigación se formula bajo la consideración que toda innovación institucional en el ámbito de la Educación Superior debería compartir esta visión sistémica, integrando y conjugando las distintas dimensiones propuestas. Además, bajo esta perspectiva, la participación estudiantil destaca como un elemento clave de la dimensión social que impacta realmente en la gobernanza universitaria.

\section{El rol del estudiante en la gobernanza universitaria}

La gobernanza universitaria ha gozado de poca atención científica, pero ha experimentado un renovado interés gracias a la creación de espacios de educación superior globales y unificados, en estas dos últimas décadas. De forma genérica, la gobernanza universitaria ha sido definida como "la manera en que las instituciones se hallan organizadas y son operadas internamente y a sus relaciones con entidades externas con vistas a asegurar los objetivos de la educación superior" (Harvey, 2004 citado por Brunner, 2011, p.139). No es el objetivo de este articulo delimitar su definición o analizar en qué modos se puede concretar, aspectos que ya han sido trabajados ampliamente por otros autores como Brunner (2011) o Ganga-Contreras et al. (2017), sino analizar el rol que juega el estudiante en esta. 
Para ello, se parte de una concepción particular de la gobernanza universitaria construida sobre los principios de democracia, autonomía y participación. Unos principios alineados con una Educación Superior que no solo busca formar profesionales competentes, sino también ciudadanos activos y comprometidos (Londres, 2007; Martínez, 2006), a la vez que impulsar el desarrollo sostenible y el liderazgo social para construir una sociedad democrática y solidaria.

Para lograr una gobernanza universitaria basada en estos tres principios, es crucial destacar la importancia de que todos los agentes de la comunidad universitaria participen en la toma de decisiones de la institución para poder articular sus distintos intereses. En este sentido, el estudiante no solo debe ser protagonista del proceso formativo, sino también copartícipe del proceso de reforma de la Educación Superior, formando parte activa del proceso de toma de decisiones institucionales. Una exigencia ampliamente formulada en los procesos de construcción de los espacios comunes de Educación Superior, tanto en el caso del Espacio Europeo de Educación Superior (Londres, 2007; Praga, 2001), como a nivel mundial (UNESCO, 1998, 2009). No obstante, esta perspectiva se opone a otras visiones vigentes de la Educación Superior, donde se concibe al estudiante esencialmente como un cliente, o se le trata de un modo paternalista (Michavila y Parejo, 2008), con lo que se convierte en un mero receptor de las políticas universitarias en vez de ser un agente activo con opiniones potencialmente útiles para la gobernanza universitaria (Merhi, 2011). En definitiva, bajo esta perspectiva, los estudiantes deben ser actores clave, tanto en la elaboración de políticas universitarias, como en la gobernanza universitaria, dándoles voz en todo el proceso (tanto en el diseño, como en la implementación y la evaluación de la política y gestión universitaria).

En cuanto al rol estudiante en la gobernanza universitaria, cabe añadir que la promoción de una cultura de participación estudiantil también tiene importantes repercusiones de carácter pedagógico; ya que configura un proceso clave para desarrollar de forma práctica algunas de las competencias cívicas propias de la Educación Superior (Martínez y Paya, 2007), a la vez que otras competencias transversales. Este aspecto es de especial relevancia en este estudio al estar focalizado en la formación de maestros, una profesión donde se esperan altos niveles competenciales y valores cívicos, a la vez que un interés profundo en los procesos e instituciones educativas como futuros profesionales de la Educación.

Sin embargo, a pesar de la versatilidad del término, la participación estudiantil suele restringirse a la participación política o representativa, o a lo sumo a la participación en políticas de calidad. Poco se habla y se ha profundizado en otros modos de participación estudiantil, como pueden ser 
el asociacionismo o la participación deportiva. Estos modos de participación no tienen una incidencia directa en la gestión o en la toma de decisiones en la gestión universitaria, pero sí que contribuyen a crear una cultura de participación, aumentado tanto el sentimiento de pertinencia de los estudiantes con la institución universitaria, como su compromiso con ella, dos factores clave para potenciar la participación representativa (Merhi, 2011; Michavila y Parejo, 2008). Además, estos otros modos de participación forman parte de la experiencia universitaria y contribuyen al desarrollo de algunas competencias y valores propios de la Educación Superior.

Por este motivo, se considera importante ampliar la noción de participación estudiantil más allá de la participación política o representativa. A continuación, se enumeran distintos modos de participación estudiantil en la universidad (Trilla et al., 2011; Ferrer y Rodero, 2012; Kouba, 2017):

- Participación académica: El estudiante toma parte activa de actividades puramente académicas. Esto puede incluir desde la asistencia regular a las clases, hasta la participación en acciones formativas complementarias o la colaboración con grupos y proyectos de investigación.

- Participación política:

- Representativa: Participación en órganos de decisión política dentro de la institución universitaria y en los procesos de gobernanza en general, a modo de representación del resto de estudiantes, normalmente por elección democrática. Esta participación puede darse en distintos niveles (incluso a nivel estatal o internacional) y mediante distintas fórmulas (vinculantes o consultivas). También incluimos la participación pasiva, relativa al acto de votar a los representantes.

- Asamblearia o sindical: Participación en espacios autoorganizados, fuera de la lógica representativa, que tienen incidencia en los procesos de toma de decisiones de la institución universitaria.

- Participación en procesos de calidad: El estudiante se convierte en un agente clave dentro del proceso evaluativo de la calidad de la Educación Superior, ya sea como consultor o como evaluador.

- Participación social: Asistencia u organización de eventos de carácter social, orientados a promover relaciones entre los propios estudiantes, ya sean de carácter puramente recreativo o con otras finalidades no académicas o políticas. Dentro de esta categoría, destaca el asociacionismo, donde un grupo de estudiantes se agrupan de forma sostenida en el tiempo para 
realizar actividades de interés común, desde ligas de debate a juegos de rol.

- Participación cultural: Asistencia u organización de eventos de carácter cultural, relacionados especialmente con las artes y el patrimonio.

- Participación deportiva: Pertenecer a equipos deportivos universitarios, o la asistencia a actividades puntuales de carácter deportivo, especialmente las organizadas dentro del contexto universitario.

- Participación comunitaria: Participación proyectada hacia el exterior de la institución universitaria. Bajo este epígrafe, podemos incluir programas de aprendizaje-servicio o proyectos de solidaridad.

- Metaparticipación: Participación resultante de la exigencia o generación de nuevos espacios y mecanismos de participación, debido a la ausencia de canales establecidos para ejercer dicha participación.

\section{Factores inhibidores y potenciadores de la participación estudiantil}

No existen datos comparativos en relación con la participación estudiantil en el contexto de América Latina. En cambio, en Europa, gracias a iniciativas como EUROSTUDENT (2000-actualidad) se ha constatado que la participación estudiantil es desigual en función del contexto geográfico, pero que, de forma general, es relativamente baja (Ariño y Llopis, 2011; Hautschild, 2015; Soler et al., 2012; Kouba, 2017). La ausencia de datos comparativos en el contexto iberoamericano dificulta hacer un diagnóstico de la situación actual que permita tomar decisiones informadas de política universitaria relativa a la participación estudiantil.

Más allá de analizar en qué grado y de qué modos participan los estudiantes universitarios, algunos estudios incipientes han investigado cuales son los factores inhibidores y potenciadores de dicha participación. Por ejemplo, Merhi (2011) afirma que la participación estudiantil requiere, cuando menos, de tres premisas: motivación (querer participar), información (saber cómo participar) y percepción de utilidad (creer que esa participación será útil). Si no se cumplen estas tres premisas, difícilmente el estudiante se implicará y comprometerá con la gobernanza universitaria. En este sentido, parece necesario realizar acciones que contribuyan a satisfacer dichas premisas; pues, por ejemplo, muchas veces existe la información, pero no llega eficazmente a los estudiantes, o es obviada debido a una sobresaturación de 
información (Merhi, 2011). En otras ocasiones, el problema puede residir en no trasmitir el valor o mostrar el impacto que tiene la participación estudiantil en la institución universitaria.

Sin embargo, para promover la participación estudiantil, no siempre es suficiente con satisfacer estas tres premisas, sino que es necesario construir una cultura de participación, que no solo debe tener lugar en la universidad, sino que además se encuentre enmarcada en la cultura de participación social (Trilla et al., 2011). De este modo, si la institución universitaria se encuentra en un contexto con una elevada participación social, será más probable que el estudiante participe dentro de su propia institución. Como ya se ha apuntado anteriormente, promover distintos modos de participación estudiantil, sin que sea necesariamente de tipo político, contribuye a la promoción de dicha cultura participativa y a aumentar la vinculación entre la universidad y los estudiantes, lo que acaba repercutiendo positivamente en un aumento de la participación en los procesos de gobernanza universitaria (Michavila y Parejo, 2008).

Además, a las tres premisas enunciadas, cabe añadir un complejo entramado de factores potenciadores e inhibidores, difícilmente identificables y desglosables. Algunos de los factores más citados y estudiados son la desafección con el sistema y los modos institucionalizados de participación (Trilla et al., 2011), la conciliación de la vida académica con la extracadémica y la laboral o familiar (Soler et al., 2012), el nulo reconocimiento de la dedicación a las tareas de representación estudiantil (Michavila y Parejo, 2008), el bajo prestigio o valor social de los modos representativos de participación política, o la falta de identificación entre el estudiante representante y el representado (Merhi, 2011). En los modos de participación no políticos, muchas veces existe un desajuste entre la oferta y la demanda de opciones de participación, lo que denota una carencia en cuanto a la participación de los propios estudiantes en los procesos de toma de decisión relativos a la oferta de actividades 0 acciones de carácter participativo.

Por este motivo, resulta imperativo llevar a cabo acciones, procesos o investigaciones de carácter sistemático que permitan obtener información relativa al contexto iberoamericano de los modos de participación existentes en las distintas universidades, del grado de participación de los estudiantes universitarios, de las motivaciones que los llevan a participar y de los motivos que han dificultado su participación, tal y como ya ocurre en otros contextos. Esta información, junto con otros indicadores y datos relativos a las condiciones de vida y estudio de los estudiantes universitarios, ha de permitir tomar decisiones informadas de política universitaria en general y, concretamente, en el ámbito de la participación estudiantil. 


\section{Método}

\subsection{Objetivos}

Se realizó un estudio cuantitativo, mediante la metodología de encuesta, centrado en explorar la dimensión social y los modos de participación universitaria de una muestra de estudiantes de programas de Educación Superior relacionados con el ámbito educativo, a través del cual se pretendía responder a las siguientes preguntas de investigación:

1. ¿Qué modos de participación estudiantil ejercen los estudiantes universitarios de programas formativos del ámbito educativo, en el contexto iberoamericano?

2. ¿Cuáles son sus motivaciones y los factores inhibidores de dicha participación?

3. ¿Existe alguna asociación entre las variables sociodemográficas de estos estudiantes y sus modos de participación dentro de la institución universitaria?

\subsection{Participantes}

La muestra total del estudio se compuso de 13939 estudiantes de grados, másteres y programas de doctorado adscritos al ámbito de las Ciencias de la Educación. En total, participaron 18 instituciones de Educación Superior de 11 países. Principalmente los participantes pertenecían a instituciones universitarias de Colombia, Argentina, Honduras, México, Ecuador, Bolivia, Paraguay, Portugal y España. A nivel de configuración sociodemográfica, la muestra estaba compuesta por 9279 mujeres $(66,6 \%)$ con una media de edad de 24,44 años (D.T. $=7,39)$ y 4660 hombres $(33,4 \%)$ con una media de edad de 24,95 años (D.T. $=7,57$ ). Tan solo un $4,6 \%$ de los participantes tenían reconocida alguna situación de diversidad funcional de cualquier tipo. El 32,4\% tenían personas dependientes a su cargo y el 8,1\% pertenecía a alguna una comunidad indígena o pueblo originario. Respecto a las variables familiares, el nivel educativo alcanzado más frecuente correspondió al de estudios básicos o secundarios, tanto en el caso del padre $(61,9 \%)$, como en el de la madre (62,7\%). Por otra parte, resultó infrecuente el hecho de que éstos no contaran con ningún tipo de estudios.

\subsection{Instrumento}

Con el fin de analizar la dimensión social de los estudiantes universitarios que cursan estudios del ámbito educativo en Iberoamérica, se creó un cuestionario compuesto por 33 ítems distribuidos en cuatro 
dimensiones: (a) datos sociodemográficos (edad, género onivel de estudios, en medidas categoriales); (b) datos académicos y satisfacción con la experiencia universitaria (estudios en curso, vía de acceso o satisfacción general, en ítems categoriales y escalares); (c) vida social y participación (situación residencial, situación laboral o modos de participación estudiantil, en ítems categoriales); y (d) ámbito motivacional (principalmente, los motivos para cursar sus estudios, en ítems escalares). Este instrumento fue validado y aporta un valor añadido gracias a su sensibilidad para ajustarse y recoger la amplia diversidad de los contextos iberoamericanos. La versión completa del cuestionario puede consultarse en Montané et al. (2019). El presente trabajo se centra en los resultados relativos a la dimensión de vida social y participación, y su relación con los datos sociodemográficos, para explicar enla mayor medida posible cómo participan los estudiantes de la muestra estudiada dentro de la institución universitaria.

\subsection{Procedimiento}

Las instituciones participantes en el proyecto TO-INN distribuyeron a sus estudiantes una versión online del cuestionario, creada a través de la plataforma Survey Monkey®, entre junio de 2018 y febrero de 2019. Antes de comenzar a cumplimentarlo, los participantes tenían que rellenar un formulario de consentimiento informado, en el que declaraban participar de forma voluntaria en el estudio, y mediante el cual el equipo investigador se comprometía a tratar la información aportada únicamente con fines de investigación, de acuerdo con la legislación internacional vigente en materia de protección de datos de carácter personal.

\subsection{Análisis de datos}

Los análisis estadísticos se realizaron mediante el software Statistical Package for Social Sciences (SPSS, versión 22) para Windows, y consistieron en la obtención de indicadores descriptivos, como el análisis de frecuencias (en el caso de los datos categóricos) o el cálculo de medias en caso de los ítems escalares, mediante el procedimiento Frecuencias, Medias y Tablas de contingencia de este software. Los ítems relativos a los distintos modos de participación universitaria fueron dicotomizados (participa/no participa). Finalmente, para contrastar la existencia de diferencias significativas entre estos ítems de participación universitaria y las diversas variables sociodemográficas, se empleó el cálculo de $X^{2}$ (Chi cuadrado), con su correspondiente significación estadística. 


\section{Resultados}

\subsection{Participación de los estudiantes dentro de la institución universitaria}

Del total de encuestados, el 51,9\% indicó haber ejercido algún modo de participación estudiantil. No obstante, un análisis desglosado sugiere que el nivel de participación fue realmente bajo en todos los modos de participación evaluados en el estudio, con una media de los porcentajes absolutos del 8,55\%. La tabla 1 resume qué modos de participación estudiantil habían ejercido aquellos estudiantes que indicaron haber participado de algún modo dentro de la institución. Cabe considerar este ítem es de opción múltiple, hecho por el cual los porcentajes que se presentan son independientes y no suman entre ellos el $100 \%$. Por ello, se debe analizar la tendencia de la participación en términos absolutos.

Tabla 1. Modos de participación estudiantil dentro de la institución universitaria

\begin{tabular}{lc}
\hline \multicolumn{1}{c}{ Modos de participación estudiantil } & Muestra participantes \\
\hline Votar en las elecciones de representantes estudiantiles & $2709(19,4 \%)$ \\
\hline Grupos artísticos o de deporte & $2317(16,6 \%)$ \\
\hline Manifestaciones, huelgas... & $1877(13,5 \%)$ \\
\hline Voluntariado (promovido por la Universidad) & $1529(11 \%)$ \\
\hline Grupos de investigación o innovación & $1303(9,3 \%)$ \\
\hline Asamblea y/o sindicato de estudiantes & $813(5,8 \%)$ \\
\hline Delegado/a de curso & $777(5,6 \%)$ \\
\hline Asociaciones estudiantiles & $723(5,2 \%)$ \\
\hline Representante estudiantil en algún órgano de gobierno & $422(3 \%)$ \\
\hline o comisión) de la Facultad/Escuela Universitaria & $383(2,7 \%)$ \\
\hline Representante estudiantil en algún órgano de gobierno & $261(1,9 \%)$ \\
\hline O comisión) general de la Universidad & \\
\hline Otras (congresos, actividades culturales, becas,...) & \\
\hline
\end{tabular}

Fuente: elaboración propia.

En cuanto a los motivos para participar en la gobernanza universitaria (tabla 2), se observó que los motivos más habituales fueron los de tipo utilitario para la persona, siendo el hecho de querer "desarrollarse a nivel personal y profesional" el motivo más frecuente, con un 49,9\% de respuestas. 
Tabla 2. Motivos para la participación universitaria

\begin{tabular}{lc}
\hline \multicolumn{1}{c}{ Motivo de participación } & Muestra participantes \\
\hline Desarrollarme a nivel personal y profesional & $3271(49,9 \%)$ \\
\hline Sentirme útil y ayudar a los demás & $676(10,3 \%)$ \\
\hline Colaborar en la mejora de la Universidad & $616(9,4 \%)$ \\
\hline Sentirme parte de la Universidad & $543(8,3)$ \\
\hline Conocer y relacionarme con otras personas & $535(8,2 \%)$ \\
\hline Tener mi propia voz en la en la organización de la Universidad & $425(6,5 \%)$ \\
\hline $\begin{array}{l}\text { Realizar actividades extraacadémicas con reconocimiento } \\
\text { curricular }\end{array}$ & $329(5 \%)$ \\
\hline Conocer de cerca el funcionamiento de la Universidad & $156(2,4 \%)$
\end{tabular}

Fuente: elaboración propia.

Finalmente, en cuanto al $48,1 \%$ de estudiantes que indicaron no haber participado de ningún modo, sus motivos para no hacerlo fueron, por orden: falta de tiempo (48\%), falta de información (23,8\%), falta de interés $(13,6 \%)$, falta de oferta por parte de la universidad $(10,8 \%)$ y llevar poco tiempo en la universidad $(3,8 \%)$, entre otros.

\subsection{Participación de los estudiantes en otros espacios asociativos fuera de la institución universitaria}

EI 55,9\% de los participantes indicó que nunca había participado en actividades asociativas fuera de la institución universitaria. La tabla 3 resume en qué ámbitos se dio esa participación en los estudiantes que sí declararon haber participado en este ámbito. Cabe considerar, de nuevo, que el ítem era de opción múltiple, hecho por el cual los porcentajes son independientes y no suman entre ellos el 100\%.

Tabla 3. Participación en actividades asociativas fuera de la institución universitaria

\begin{tabular}{lc}
\hline \multicolumn{1}{c}{ Actividad asociativa } & Muestra participantes \\
\hline Asociaciones o clubes deportivos & $1699(12,2 \%)$ \\
\hline Organizaciones juveniles & $1679(12 \%)$ \\
\hline ONG y asociaciones de voluntariado & $1676(12 \%)$ \\
\hline Asociaciones culturales & $1547(11,1 \%)$ \\
\hline Asociaciones o grupos religiosos & $1486(10,7 \%)$ \\
\hline Partidos políticos & $888(6,4 \%)$ \\
\hline Asociaciones de vecinos & $416(3 \%)$ \\
\hline Sindicatos profesionales & $283(2 \%)$ \\
\hline
\end{tabular}

Fuente: elaboración propia. 


\subsection{Variables sociodemográficas y modos de participación estudiantil}

A continuación, se presentan los resultados del análisis de la asociación entre la participación estudiantil y las variables sociodemográficas más relevantes: género, edad, diversidad funcional, pertenencia a comunidades indígenas, tener personas a su cargo y nivel formativo familiar.

En primer lugar, con relación al género, existen diferencias significativas en la mayoría de los modos de participación estudiantil (tabla 4), siendo los estudiantes masculinos los que tendieron a participar más en la mayoría de los ámbitos evaluados.

Tabla 4. Relación entre los modos de participación estudiantil y el género

\begin{tabular}{lcr}
\hline \multicolumn{1}{c}{ Modos de participación estudiantil } & $\begin{array}{c}\text { Relación con el } \\
\text { género }\end{array}$ & Mayor participación \\
\hline $\begin{array}{l}\text { Votar en las elecciones de representantes } \\
\text { estudiantiles }\end{array}$ & $\mathrm{X}^{2}=88,65 ; \mathrm{p}<, 005$ & Género masculino \\
\hline Grupos artísticos o de deporte & $\mathrm{X}^{2}=29,05 ; \mathrm{p}<, 005$ & Género masculino \\
\hline Manifestaciones, huelgas... & $\mathrm{X}^{2}=20,32 ; \mathrm{p}<, 005$ & Género masculino \\
\hline Voluntariado (promovido por la Universidad) & $\mathrm{X}^{2}=4,76 ; \mathrm{p}=, 031$ & Género masculino \\
\hline Grupos de investigación o innovación & No significativa & Similar \\
\hline Asamblea y/o sindicato de estudiantes & No significativa & Similar \\
\hline $\begin{array}{l}\text { Delegado/a de curso } \\
\text { Asociaciones estudiantiles }\end{array}$ & $\mathrm{X}^{2}=135,54 ; \mathrm{p}<, 005$ & Género masculino \\
\hline $\begin{array}{l}\text { Representante estudiantil en algún órgano de } \\
\text { gobierno (o comisión) de la Facultad/Escuela } \\
\text { Universitaria }\end{array}$ & $\mathrm{X}^{2}=34,36 ; \mathrm{p}<, 005$ & Género masculino \\
\hline $\begin{array}{l}\text { Representante estudiantil en algún órgano } \\
\text { de gobierno (o comisión) general de la } \\
\text { Universidad }\end{array}$ & No significativa & Género masculino \\
\hline Fuente: & & Similar \\
\hline
\end{tabular}

Fuente: elaboración propia.

En cuanto a la edad, su relación con los modos de participación estudiantil resultó estadísticamente significativa (tabla 5). En este sentido, se detectaron tres tendencias diferentes: (a) Las personas más jóvenes (menos de 23 años) manifestaron una mayor participación deportiva o artística; (b) Las personas más mayores (más de 30 años) se implicaron más con la representación política, ya sea en órganos de gobierno generales de la Universidad o de la propia Facultad, o bien a modo de delegados de curso, a la vez que con los grupos de investigación o innovación; y (c) Los estudiantes situados en la franja entre 23 y 30 años se implicaron más que sus compañeros a la 
hora de votar en las elecciones de representantes estudiantiles, en la participación asamblearia o sindical, en las asociaciones de estudiantes, en la realización de manifestaciones y huelgas, y en la participación en acciones de voluntariado.

Tabla 5. Relación entre los modos de participación estudiantil y la edad.

\begin{tabular}{|c|c|c|}
\hline Modos de participación estudiantil & Relación con la edad & Mayor participación \\
\hline $\begin{array}{l}\text { Votar en las elecciones de representantes es- } \\
\text { tudiantiles }\end{array}$ & $X^{2}=95,71 ; p<$, & Entre 23 y 30 años \\
\hline Grupos artísticos o de deporte & $X^{2}=93,32 ; p<, 005$ & Menos de 23 años \\
\hline Manifestaciones, huelgas... & $x^{2}=133,16 ; p<, 005$ & Entre 23 y 30 años \\
\hline Voluntariado (promovido por la Universidad) & $X^{2}=102,28 ; p<, 005$ & Entre 23 y 30 años \\
\hline Grupos de investigación o innovación & $X^{2}=85,59 ; p<, 005$ & Más de 30 años \\
\hline Asamblea y/o sindicato de estudiantes & $X^{2}=42,45 ; p<, 005$ & Entre 23 y 30 años \\
\hline Delegado/a de curso & $X^{2}=25,23 ; p<, 005$ & 30 años \\
\hline Asociaciones estudiantiles & $X^{2}=13,25 ; p<, 005$ & Entre 23 y 30 años \\
\hline $\begin{array}{l}\text { Representante estudiantil en algún órgano de } \\
\text { gobierno (o comisión) de la Facultad/Escuela } \\
\text { Universitaria }\end{array}$ & $X^{2}=38,07 ; p<, 005$ & Más de 30 años \\
\hline $\begin{array}{l}\text { Representante estudiantil en algún órgano de } \\
\text { gobierno (o comisión) general de la Universidad }\end{array}$ & $X^{2}=85,59 ; p<, 005$ & Más de 30 años \\
\hline
\end{tabular}

Fuente: elaboración propia.

En cuanto a la pertenencia a comunidades indígenas (tabla 6), se observó que el hecho de pertenecer a este tipo de comunidades implica una menor participación política, ya sea a la hora de votar en las elecciones de representantes estudiantiles, o como representantes estudiantiles en órganos de gobierno generales de la Universidad, en asambleas o en sindicatos de estudiantes.

Al mismo tiempo, estos estudiantes acudieron menos a manifestaciones o huelgas y participan menos en acciones de voluntariado y en grupos de investigación o innovación. En el resto de los ámbitos de participación no se observaron diferencias estadísticamente significativas. Para profundizar en las características sociodemográficas y las condiciones de vida y estudio de los estudiantes de educación de origen indígena, vean nuestro artículo publicado en la RASE (Méndez et al., 2019). 
Tabla 6. Relación entre los modos de participación estudiantil y la pertenencia a comunidades indígenas.

\begin{tabular}{|c|c|c|}
\hline Modos de participación estudiantil & $\begin{array}{c}\text { Relación con la } \\
\text { pertenencia a } \\
\text { comunidades indígenas }\end{array}$ & Mayor participación \\
\hline $\begin{array}{l}\text { Votar en las elecciones de representantes } \\
\text { estudiantiles }\end{array}$ & No significativa & Similar \\
\hline Grupos artísticos o de deporte & No significativa & Similar \\
\hline Manifestaciones, huelgas... & $x^{2}=94,75 ; p<, 005$ & $\begin{array}{l}\text { Estudiantes sin } \\
\text { origen indígena }\end{array}$ \\
\hline $\begin{array}{l}\text { Voluntariado (promovido por la } \\
\text { Universidad) }\end{array}$ & $X^{2}=6,09 ; p<, 005$ & $\begin{array}{l}\text { Estudiantes sin } \\
\text { origen indígena }\end{array}$ \\
\hline Grupos de investigación o innovación & $x^{2}=7,42 ; p<, 005$ & $\begin{array}{l}\text { Estudiantes sin } \\
\text { origen indígena }\end{array}$ \\
\hline Asamblea y/o sindicato de estudiantes & $X^{2}=57,22 ; p<, 005$ & $\begin{array}{l}\text { Estudiantes sin } \\
\text { origen indígena }\end{array}$ \\
\hline Delegado/a de curso & No significativa & Similar \\
\hline Asociaciones estudiantiles & No significativa & Similar \\
\hline $\begin{array}{l}\text { Representante estudiantil en algún } \\
\text { órgano de gobierno (o comisión) de la } \\
\text { Facultad/Escuela Universitaria }\end{array}$ & No significativa & Similar \\
\hline $\begin{array}{l}\text { Representante estudiantil en algún } \\
\text { órgano de gobierno (o comisión) general } \\
\text { de la Universidad }\end{array}$ & $X^{2}=11,71 ; p<, 005$ & $\begin{array}{l}\text { Estudiantes sin } \\
\text { origen indígena }\end{array}$ \\
\hline
\end{tabular}

Fuente: elaboración propia.

Por otra parte, los resultados obtenidos sugieren que el hecho de tener personas a cargo tiene una implicación directa en los modos de participación estudiantil (tabla 7), pues este colectivo participó menos a la hora de votar en las elecciones de representantes estudiantiles o como representantes estudiantiles en órganos de gobierno generales de su universidad, en asambleas o en sindicatos de estudiantes, a la vez que acudieron menos a manifestaciones o huelgas. Además, participaron en menor grado en acciones de voluntariado y en grupos artísticos o deportivos. El resto de los modos de participación no mostraron diferencias estadísticas significativas.

Tabla 7. Relación entre los modos de participación estudiantil y el hecho de tener personas a cargo

\begin{tabular}{lrl}
\hline \multicolumn{1}{c}{ Modos de participación estudiantil } & $\begin{array}{r}\text { Relación con el } \\
\text { hecho de tener } \\
\text { persona a cargo }\end{array}$ & Mayor participación \\
\hline $\begin{array}{l}\text { Votar en las elecciones de representantes es- } \\
\text { tudiantiles }\end{array}$ & $\mathrm{X}^{2}=50,74 ; \mathrm{p}<, 005$ & $\begin{array}{l}\text { Estudiantes } \\
\text { sin personas a cargo }\end{array}$ \\
\hline Grupos artísticos o de deporte & $\mathrm{X}^{2}=24,05 ; \mathrm{p}<, 005$ & $\begin{array}{l}\text { Estudiantes } \\
\text { sin personas a cargo }\end{array}$ \\
\hline Manifestaciones, huelgas... & $\mathrm{X}^{2}=216,50 ; \mathrm{p}<, 005$ & $\begin{array}{l}\text { Estudiantes } \\
\text { sin personas a cargo }\end{array}$ \\
\hline
\end{tabular}




\begin{tabular}{|c|c|c|}
\hline Modos de participación estudiantil & $\begin{array}{l}\text { Relación con el } \\
\text { hecho de tener } \\
\text { persona a cargo }\end{array}$ & Mayor participación \\
\hline Voluntariado (promovido por la Universidad) & $X^{2}=15,44 ; p<, 005$ & $\begin{array}{l}\text { Estudiantes } \\
\text { sin personas a cargo }\end{array}$ \\
\hline Grupos de investigación o innovación & No significativa & Similar \\
\hline Asamblea y/o sindicato de estudiantes & $X^{2}=35,48 ; p<, 005$ & $\begin{array}{l}\text { Estudiantes } \\
\text { sin personas a cargo }\end{array}$ \\
\hline Delegado/a de curso & No significativa & Similar \\
\hline Asociaciones estudiantiles & No significativa & Similar \\
\hline $\begin{array}{l}\text { Representante estudiantil en algún órgano de } \\
\text { gobierno (o comisión) de la Facultad/Escuela } \\
\text { Universitaria }\end{array}$ & No significativa & Similar \\
\hline $\begin{array}{l}\text { Representante estudiantil en algún órgano de } \\
\text { gobierno (o comisión) general de la Universidad }\end{array}$ & $X^{2}=4,88 ; p=, 021$ & $\begin{array}{l}\text { Estudiantes } \\
\text { sin personas a cargo }\end{array}$ \\
\hline
\end{tabular}

Fuente: elaboración propia.

Además, los resultados obtenidos sugieren que el hecho de tener una discapacidad o situación de diversidad funcional reconocida condiciona los modos de participación de este colectivo (tabla 8), reduciéndola en la mayoría de los ámbitos; excepto en la representación en órganos de gobierno (o comisiones) generales de la universidad, donde indicaron una mayor participación en relación con el resto de los estudiantes encuestados.

Tabla 8. Relación entre los modos de participación estudiantil y la condición de diversidad funcional

\begin{tabular}{|c|c|c|}
\hline Modos de participación estudiantil & $\begin{array}{l}\text { Relación con } \\
\text { la condición de } \\
\text { discapacidad }\end{array}$ & Mayor participación \\
\hline $\begin{array}{l}\text { Votar en las elecciones de representan- } \\
\text { tes estudiantiles }\end{array}$ & $X^{2}=79,44 ; p<, 005$ & Estudiantes sin discapacidad \\
\hline Grupos artísticos o de deporte & $x^{2}=66,42 ; p<, 005$ & Estudiantes sin discapacidad \\
\hline Manifestaciones, huelgas... & $x^{2}=54,52 ; p<, 005$ & Estudiantes sin discapacidad \\
\hline $\begin{array}{l}\text { Voluntariado (promovido por la Uni- } \\
\text { versidad) }\end{array}$ & $x^{2}=13$ & Estudiantes sin disca \\
\hline Grupos de investigación o innovación & $X^{2}=38,94 ; p<, 005$ & Estudiantes sin discapacidad \\
\hline Asamblea y/o sindicato de estudiantes & $X^{2}=12,55 ; p<, 005$ & Estudiantes sin discapacidad \\
\hline Delegado/a de curso & $X^{2}=17,84 ; p<, 005$ & Estudiantes sin discapacidad \\
\hline Asociaciones estudiantiles & $X^{2}=5,43 ; p=, 022$ & Estudiantes sin discapacidad \\
\hline $\begin{array}{l}\text { Representante estudiantil en algún } \\
\text { órgano de gobierno (o comisión) de la } \\
\text { Facultad/Escuela Universitaria }\end{array}$ & No significativa & Similar \\
\hline $\begin{array}{l}\text { Representante estudiantil en algún } \\
\text { órgano de gobierno (o comisión) general } \\
\text { de la Universidad }\end{array}$ & $X^{2}=490,12 ; p<, 005$ & Estudiantes con discapacidad \\
\hline
\end{tabular}


Finalmente, en relación con el nivel formativo familiar, se hallaron diferencias estadísticamente significativas, siendo los estudiantes que provienen de un entorno familiar con estudios superiores o con formación profesional normalmente los que más participaron en la vida universitaria. A continuación, se desglosan en dos tablas estos resultados en función del nivel formativo de la madre (tabla 9) y del padre (tabla 10).

Tabla 9. Relación entre los modos de participación estudiantil y el nivel formativo de la madre

\begin{tabular}{|c|c|c|}
\hline Modos de participación estudiantil & $\begin{array}{l}\text { Relación con la ocu- } \\
\text { pación de la madre }\end{array}$ & Mayor participación \\
\hline $\begin{array}{l}\text { Votar en las elecciones de representantes es- } \\
\text { tudiantiles }\end{array}$ & $x^{2}=27,64 ; p<, 005$ & $\begin{array}{l}\text { Hijos de madres con } \\
\text { formación profesional }\end{array}$ \\
\hline Grupos artísticos o de deporte & No significativa & Similar \\
\hline Manifestaciones, huelgas... & $x^{2}=62,22 ; p<, 005$ & $\begin{array}{l}\text { Hijos de madres con } \\
\text { formación profesional }\end{array}$ \\
\hline Voluntariado (promovido por la Universidad) & $X^{2}=12,79 ; p=, 012$ & $\begin{array}{l}\text { Hijos de madres con } \\
\text { estudios superiores }\end{array}$ \\
\hline Grupos de investigación o innovación & No significativa & Similar \\
\hline Asamblea y/o sindicato de estudiantes & $X^{2}=14,08 ; p=, 007$ & $\begin{array}{l}\text { Hijos de madres con } \\
\text { formación profesional }\end{array}$ \\
\hline Delegado/a de curso & $X^{2}=18,74 ; p<, 005$ & $\begin{array}{l}\text { Hijos de madres con } \\
\text { estudios superiores }\end{array}$ \\
\hline Asociaciones estudiantiles & No significativa & Similar \\
\hline $\begin{array}{l}\text { Representante estudiantil en algún órgano de } \\
\text { gobierno (o comisión) de la Facultad/Escuela } \\
\text { Universitaria }\end{array}$ & $X^{2}=24,38 ; p<, 005$ & $\begin{array}{l}\text { Hijos de madres con } \\
\text { estudios superiores }\end{array}$ \\
\hline $\begin{array}{l}\text { Representante estudiantil en algún órgano de } \\
\text { gobierno (o comisión) general de la Universidad }\end{array}$ & $x^{2}=47,77 ; p<, 005$ & $\begin{array}{l}\text { Hijos de madres con } \\
\text { formación profesional }\end{array}$ \\
\hline
\end{tabular}

Fuente: elaboración propia.

Tabla 10. Relación entre los modos de participación estudiantil y el nivel formativo del padre

\begin{tabular}{lcl}
\hline \multicolumn{1}{c}{ Modos de participación estudiantil } & $\begin{array}{c}\text { Relación con la } \\
\text { ocupación de la } \\
\text { madre }\end{array}$ & Mayor participación \\
\hline $\begin{array}{l}\text { Votar en las elecciones de representantes es- } \\
\text { tudiantiles }\end{array}$ & $\mathrm{X}^{2}=21,85 ; \mathrm{p}<, 005,46 ; \mathrm{p}<, 005$ & $\begin{array}{l}\text { Hijos de padres con } \\
\text { formación profesional }\end{array}$ \\
\hline Grupos artísticos o de deporte & $\mathrm{X}^{2}=55,44 ; \mathrm{p}<, 005$ & $\begin{array}{l}\text { Hijos de padres con } \\
\text { formación profesional }\end{array}$ \\
\hline Manifestaciones, huelgas... & No significativa & Similar \\
\hline Voluntariado (promovido por la Universidad)
\end{tabular}




\begin{tabular}{lll}
\hline \multicolumn{1}{c}{ Modos de participación estudiantil } & $\begin{array}{c}\text { Relación con la } \\
\text { ocupación de la } \\
\text { madre }\end{array}$ & Mayor participación \\
\hline Delegado/a de curso & No significativa & Similar \\
\hline Asociaciones estudiantiles & No significativa & Similar \\
\hline $\begin{array}{l}\text { Representante estudiantil en algún órgano de } \\
\text { gobierno (o comisión) de la Facultad/Escuela }\end{array}$ & $\mathrm{X}^{2}=20,22 ; \mathrm{p}<, 005$ & Hijos de padres con \\
$\begin{array}{l}\text { Universitaria } \\
\text { Representante estudiantil en algún órgano de } \\
\text { gobierno (o comisión) general de la Universidad }\end{array}$ & $\mathrm{X}^{2}=36,47 ; \mathrm{p}<, 005$ & $\begin{array}{l}\text { Hijos de padres con } \\
\text { estudios superiores }\end{array}$ \\
\hline Fuente: elaboración propia.
\end{tabular}

Fuente: elaboración propia.

\section{Discusión}

En primer lugar, aunque la cantidad de estudiantes que indicaron haber ejercido algún modo de participación fue elevada (51,9\%), su análisis desglosado constata que la participación en general fue baja, ya que la media de los porcentajes absolutos fue del 8,55\%. Además, fue especialmente baja en cuanto a la participación política: la forma representativa se situó en un nivel de participación alrededor del 3\% y la asamblearia o sindical en el $5,2 \%$. Los resultados obtenidos se encuentran en la línea de lo que reportan otros estudios sobre participación política de los estudiantes y gobernanza universitaria en otros contextos (Ariño y Llopís, 2011; Haustschild, 2015; Soler et al., 2012). Por otro lado, resulta sorprendente el elevado porcentaje de participación en actividades reivindicativas (manifestaciones, huelgas, etc.) y de voluntariado ( $13,5 \%$ y $11 \%$, respectivamente), siendo significativamente superior a la reportada en otros contextos como el europeo o norteamericano. Estos resultados se podrían relacionar con cuestiones socioculturales de la comunidad iberoamericana y con el fuerte desarrollo de la relación universidad-sociedad que se ha dado desde hace tiempo en las instituciones de Educación Superior iberoamericanas.

Al analizar los motivos alegados para no participar en la vida y gestión universitaria, estos hacen referencia a los principales elementos inhibidores señalados por la literatura previa: falta de tiempo (48\%), de información $(23,8 \%)$ y de interés $(13,6 \%)$. Esto reafirma la necesidad de trabajar en el fomento de políticas universitarias de participación estudiantil que den respuesta a las tres premisas indicadas por Merhi (2011): motivación, (in) formación y utilidad percibida. A su vez, cabe atender a la falta de tiempo, pues, aunque no conocemos los motivos exactos, sí que existen indicadores que señalan las dificultades de compaginar la participación extracadémica con la académica, o de conciliarla con la vida laboral y personal. Por ejemplo, se 
observó que los estudiantes con personas dependientes a su cargo participan en menor medida que los demás. En este sentido, es necesario pensar cómo adaptar la participación estudiantil para dar respuesta a las necesidades y condiciones específicas de estos colectivos.

Finalmente, en cuanto a la participación general del colectivo estudiantil, resulta oportuno señalar que la participación política en órganos de representación, tanto general como en las Facultades, es casi la mitad que la participación política de carácter asambleario o sindical, lo cual refleja la desafección por los modelos formales establecidos por la institución de representación y la búsqueda de espacios más propios y autorganizados. En este sentido, la participación asamblearia o sindical es superior. Estos datos están en consonancia con la desafección con el sistema por parte de los estudiantes, expresada por Trilla et al. (2011), o el bajo prestigio o valor desolar de los modos representativos de participación política, y la falta de identificación entre el estudiante representante y el estudiante representado, a la que alude Merhi (2011).

Además, los resultados obtenidos sugieren que en el contexto iberoamericano también hay una baja participación en actividades asociativas fuera de la institución universitaria, pues ninguno de los modos de participación superó en nuestro estudio el 12,2\%. Promover la participación social fuera de la universidad podría contribuir a aumentar la participación estudiantil dentro de la institución universitaria, tal y como ya han apuntado algunos autores como Trilla et al. (2011).

Queda demostrado que la participación estudiantil es uno de los elementos de la vida universitaria que se encuentra fuertemente influenciado por las características sociodemográficas. En este sentido, existen algunos condicionantes que reducen de forma general la participación de los estudiantes: la pertenencia a comunidades indígenas, el hecho de tener personas a cargo o la condición de discapacidad o diversidad funcional. En estas tres situaciones, los estudiantes tienden a participar menos en la vida universitaria, excepto curiosamente en el caso de las personas con discapacidad, que tienden a participar más como 'representantes estudiantiles en algún órgano de gobierno (o comisión) de la Facultad/Escuela Universitaria' que el resto de los estudiantes. De este modo, igual que ocurre en el acceso a la universidad, la participación estudiantil tampoco cumple el principio de equidad participativa (Londres, 2007). Es decir, se produce una infrarrepresentación de ciertos colectivos en determinados modos de participación estudiantil, tanto respeto a la población universitaria, como de la población general. 
La participación tampoco fue equitativa en nuestra muestra con relación al género, pues de forma generalizada, los estudiantes varones participaron más que sus compañeras. De cara al futuro, es importante indagar en los motivos de este fenómeno; para saber si responde a cuestiones estructurales o motivacionales. También se observaron unos niveles de participación desigual en función de la edad, pues precisamente el grupo de estudiantes menos numeroso, el de estudiantes maduros (más de 30 años), son los que más participaron de forma política tanto a modo de delegados, como mediante fórmulas representativas, lo que indica una mayor implicación con la gobernanza universitaria.

Finalmente, los resultados obtenidos aportan una visión renovada en cuanto a la relación entre la participación estudiantil y el nivel formativo familiar. A este respecto, se observó que el nivel formativo de la madre y del padre podrían tener una cierta influencia sobre los modos de participación en que se involucran los estudiantes universitarios. En este sentido, los estudiantes que provienen de un entorno familiar con estudios de formación profesional suelen participar más a nivel deportivo y reivindicativo, a la vez que en la participación política pasiva y asamblearia o sindical. En cambio, los estudiantes que provienen de un entorno familiar con estudios superiores suelen presentar un mayor nivel de participación como delegados de curso y en formas de participación política representativa que el resto de sus compañeros.

\section{Conclusiones}

La participación estudiantil dentro de la institución universitaria es baja en el contexto iberoamericano, igual que ocurre en otros contextos, aunque es ligeramente superior en cuanto a la participación en acciones reivindicativas y de voluntariado. En este sentido, la promoción de la participación estudiantil sigue siendo uno de los retos de la institución universitaria actual, especialmente si se pretende lograr una gobernanza universitaria basada en los principios de democracia, autonomía y participación. Solo así, el estudiante podrá convertirse realmente en un agente clave, tanto en la gestión como en el diseño de políticas universitarias.

Para ello, es fundamental trabajar en políticas de participación que den respuesta a las siguientes premisas: motivación, (in)formación y utilidad percibida, junto con modelos de participación que permitan su conciliación con la vida académica, la laboral y la personal. Otras de las medidas que podrían resultar oportunas para impulsar la participación estudiantil son: el reconocimiento de la participación a nivel curricular; la promoción de la cultura participativa a nivel social y en niveles educativos previos; la comu- 
nicación de su utilidad en cuanto a la mejora institucional o al aprendizaje informal de competencias; o la elaboración de políticas universitarias que se ocupen de la dimensión social de la universidad.

Por otro lado, los resultados obtenidos constatan la existencia de diferencias significativas en los modos de participar de ciertos colectivos de estudiantes, como pueden ser los estudiantes mayores de 30 años, los estudiantes en situación de diversidad funcional, con personas dependientes a cargo o los estudiantes pertenecientes a comunidades indígenas. En general, pareciera que es necesario adaptar y repensar algunos modos de participación estudiantil para dar respuesta a las necesidades y condiciones específicas de algunos colectivos, del mismo modo que desde hace tiempo se habla de flexibilizar los espacios de aprendizaje para dichos colectivos.

Este estudio constituye una primera investigación sistemática y comparada con instituciones de distintos países del contexto iberoamericano sobre las condiciones de vida y de estudio de los estudiantes universitarios, concretamente del ámbito de las Ciencias de la Educación. Sin embargo, en cuanto a las limitaciones del estudio, cabe señalar la necesidad de explorar en mayor profundidad los motivos subyacentes a dichos modos de participación, mediante propuestas metodológicas mixtas o cualitativas. Además, es necesario no obviar la dificultad de elaborar conclusiones generales derivadas de un estudio de carácter comparativo como este, debido a la amplia diversidad sociopolítica de las instituciones participantes y de sus sistemas educativos. En definitiva, este estudio constituye un punto de partida para otras iniciativas orientadas a conocer con mayor profundidad quiénes y cómo son los estudiantes universitarios en el contexto iberoamericano, a la vez de sus modos de participación, para poder tomar decisiones de política universitaria; no sólo del ámbito de las Ciencias de la Educación, sino también en el resto de los ámbitos de conocimientos.

\section{Bibliografía}

Antúnez, S. (2019). Hacia la mejora de la Gobernanza en las Instituciones de Educación Superior de Formación Docente. En S. Antúnez y A. Montané (Eds.), Política y Gobernanza en las Instituciones de Educación Superior de Formación Docente, pp. 5-21. Institut de Creativitat i Innovacions Educatives de la Universitat de València.

Ariño, A. (2014). La dimensión social en la educación superior. Revista de la Asociación de Sociología de la Educación, 7(1), 17-41.

Ariño, A. y Llopis, R. (2011). ¿Universidad sin clases? Condiciones de vida de los estudiantes universitarios en España (Eurostudent IV). Secretaría General Técnica, MECD. 
Brunner, J. J. (2011). Gobernanza universitaria: tipología, dinámicas y tendencias. Revista de Educación, 355, 137-159.

Ferrer, M. y Rodero, E. (2012). La participación estudiantil en la UPF. Universitat Pompeu Fabra. https://bit.ly/2x6dxR0

Ganga-Contreras, F., Quiroz, J. y Fossatti, P. (2017). Análisis sincrónico de la gobernanza universitaria: una mirada teórica a los años sesenta y setenta. Educaçao e Psiquisa, 43(2), 553-568. https://doi.org/10.1590/s1517-9702201608135289

Hautschild, K. (Ed.) (2015). Social and Economic Conditions of Student Life in Europe. EUROSTUDENT V, 2012-2015. Synopsis of Indicators. W. Bertelsmann Verlag GmbH \& Co. KG. https://doi.org/10.3278/6001920bw

Kouba, K. (2017). Determinants of student participation in higher education governance: the case of student turnout in academics en ate elections in Czechia. Higher Education, 76(1), 67-84. https://doi.org/10.1007/s10734-017-0194-1

Londres, Comunicado de (2007). Towards the European Higher Education Area: responding to challenges in a globalised world. https://bit.ly/3abTI92

Martínez, M. (2006). Formación para la ciudadanía y Educación Superior. Revista Iberoamericana de Educación, 42, 85-102.

Martínez, M. y Payá, M. (Coords.) (2007). La formación de la ciudadanía en el Espacio Europeo de Educación Superior. En J. L. García Garrido (Ed.), Formar ciudadanos europeos (pp. 58-80). Academia Europea de Ciencias y Artes.

Méndez, J., Montané, A., Llanes, J., Hervas, G., Calduch, I., Morales-Ulloa, R. y Muñoz, J. (2019). Características sociodemográficas y condiciones de vida y estudio de los estudiantes de Educación de origen indígena. La aproximación del proyecto TO-INN. Revista de Sociología de la Educación, 12(2), 139-156.

Merhi, R. (2011). Las claves de la participación estudiantil en la universidad española [Comunicación en congreso]. Congreso Internacional Univest 2011, Girona, España.

Michavila, F. y Parejo, J. L. (2008). Políticas de participación estudiantil en el Proceso de Bolonia. Revista de Educación, número extraordinario, 85-118.

Montané, A., Llanes, J., Calduch, I., Hervás, G., Méndez, J. y Muñoz, J. (2019). Dimensión social y universidad. Diseño y aplicación de un instrumento para conocer a los estudiantes universitarios. En A. Bon y M. Pini (Eds.), Cultura, Ciudadanía, Participación. Perspectivas de la Educación Inclusiva, pp. 141-170. Pangea.

OEI (2010). Metas Educativas 2021: La educación que queremos para la generación de los bicentenarios. Organización de Estados Iberoamericanos: Madrid.

ONU (2018). Resolución de la Asamblea General “Transformar nuestro mundo: la Agenda 2030 para el Desarrollo Sostenible" A/RES/70/1 (25 de septiembre de 2015). https:// bit.ly/3cjw4ZW

Praga, Comunicado de (2001). Hacia el Área de la Educación Superior Europea. https://bit. $\underline{\text { ly/2wbQaVy }}$

Soler, P., Pallisera, M., Planas, A., Fullana, J. y Vilà, M. (2012). La participación de los estudiantes en la universidad: dificultades percibidas y propuestas de mejora. Revista de Educación, 358, 542-562. 
Trilla, J., Jover, G., Martínez, M. y Romañá, T. (2011). La participación de los estudiantes en el gobierno y la vida universitaria. Encounters on Education, 12, 93-111.

UNESCO (1998). Declaración mundial sobre la educación superior en el siglo XXI: visión y acción. UNESCO. https://bit.ly/2uCc2sH

UNESCO (2009). Conferencia Mundial sobre la Educación Superior 2009: La nueva dinámica de la Educación Superior y la investigación para el cambio social y el desarrollo. UNESCO. https://bit.ly/2/1GM9x

UNESCO (2015). Rethinking Education: Towards a global common good? UNESCO. 